\title{
Midbrain Hypometabolism in Fatal Familial Insomnia: A Case Report and a Statistical Parametric Mapping Analysis of a Korean Family
}

\author{
Mi Ji Lee ${ }^{a}$ Jisoo Shin ${ }^{a}$ Eun Joo Chung ${ }^{c}$ Sang-Jin Kim ${ }^{c}$ \\ Soonwook Kwon ${ }^{\mathrm{a}}$ Jung-Hyun Kim ${ }^{\mathrm{a}}$ Sang Won Seo \\ Chang-Seok Ki ${ }^{b}$ Duk L. Na ${ }^{a}$ \\ Departments of ${ }^{a}$ Neurology and ${ }^{b}$ Laboratory Medicine and Genetics, Samsung Medical \\ Center, Sungkyunkwan University School of Medicine, Seoul, and 'Department of \\ Neurology, Busan Paik Hospital, Inje University College of Medicine, Busan, \\ Republic of Korea
}

\section{Key Words}

Fatal familial insomnia - Glucose hypometabolism · Statistical parametric mapping analysis

\begin{abstract}
Background and Methods: Fatal familial insomnia (FFI) is a rare genetic disease characterized by intractable insomnia, dysautonomia, and dementia. Herein we describe a patient with FFI. In order to study brain glucose hypometabolism in the patient, we used statistical parametric mapping (SPM) analysis of $\left[{ }^{18} \mathrm{~F}\right]$-fluorodeoxyglucose positron emission tomography (FDG-PET). Case Report: The patient was a 34-year-old Korean man. He presented with intractable insomnia, rapidly progressive dementia and autonomic disturbances. A comprehensive clinical investigation was conducted, including brain MRI, electroencephalography, polysomnography, neuropsychological tests, FDG-PET and genomic tests. SPM analysis was performed using 7 healthy controls. Direct sequencing of the PRNP gene identified a heterozygous p.Asp179Asn mutation homozygous for methionine at codon 129 and for glutamate at codon 219. The results of the SPM analysis showed marked hypometabolism in the deep cerebral nuclei (including the bilateral thalami, caudate nuclei, and hypothalamus), association cortices (including the frontal, lateral temporal, inferior parietal lobule and posterior cingulate gyri), and midbrain. Conclusions: This is the first Korean report of FFI, in which the family showed male phenotypic predominance. The patient's SPM analysis demonstrated brain hypometabolism in the midbrain and the hypothalamus, as well as the
\end{abstract}


thalami, caudate nuclei, and multiple cortical regions. These results contribute further to the overall understanding of the pathophysiology of FFI.

(c) 2014 S. Karger AG, Basel

\section{Introduction}

Fatal familial insomnia (FFI) is a rare disease characterized by intractable insomnia, dysautonomia, and mental deterioration [1]. FFI is classified as a genetically transmitted human transmissible spongiform encephalopathy [1]. Point mutation at codon 178 of PRNP is the diagnostic genetic abnormality of FFI.

We herein report a patient with FFI, who, to our knowledge, is the first case in the Korean population. In addition to a detailed history and the results of comprehensive neuropsychological tests, we also present the patient's statistical parametric mapping (SPM) analysis of [18F]-fluorodeoxyglucose positron emission tomography (FDG-PET) compared with 7 agematched normal controls.

\section{Case Report}

\section{Clinical Description}

The patient was a 34-year-old Korean man who visited our emergency department complaining of intractable insomnia and progressive dementia. The patient suffered from intractable insomnia and irregular, coarse breathing during sleep, which began 9 months before admission. He consequently developed excessive sweating, bilateral action tremors, and restlessness. Memory disturbance and poor executive function were noticed 2 months before admission. Delusions of persecution and social withdrawal were also present. Three days before admission, the patient began to show gait disturbance with mild postural instability.

The patient had been in good health before disease onset. He had 16 years of education, majored in law, and worked as a civil servant at the district court until he lost his job due to memory disturbance.

His vital signs showed a marked fluctuation in blood pressure, tachycardia, and tachypnea. His initial body temperature was $38.0^{\circ} \mathrm{C}$, which was stabilized after controlling his pneumonia.

Neurologic examination revealed disorientation and anomic aphasia with paraphasia. The Korean version of the Mini-Mental State Examination score was 17 out of 30. Hypophonia, dysarthria, both resting and action tremors, and akathisia were present. His gait was ataxic with a tendency of tilting backwards. Otherwise, cranial nerve functions, muscle volume, tone, and power as well as sensory functions were normal. Extrapyramidal signs such as rigidity or bradykinesia were absent. Deep tendon reflexes were normoactive without pathologic reflexes.

Routine blood labs showed no abnormalities. Both plasma and urine catecholamine levels were elevated. Cerebrospinal fluid analysis was unremarkable and the patient was negative for 14-3-3 protein.

\section{Family History}

The patient's four-generation pedigree is presented in fig. 1. The patient's mother (III:7), who was a physically and mentally healthy 70 -year-old woman, had the same mutation. An asymptomatic brother of the proband (IV:12) was negative for the PRNP gene mutation. The 
son of the patient's great-aunt (II:1) had gradually developed hypersomnia, gait disturbance, ataxia, and dysarthria. His cerebrospinal fluid study was positive for 14-3-3 protein. The genetic analysis of subject II:1 revealed a PRNP gene mutation at codon 178 (D178N) and homozygosity for methionine at codon 129. Total disease duration was 27 months.

\section{Clinical Investigations}

Magnetic resonance imaging and electroencephalography findings were unremarkable. Polysomnography revealed reduced total sleep time, absence of slow-wave and REM stages of sleep, severe periodic limb movement, and heavy snoring.

\section{Neuropsychological Studies}

Neuropsychological tests were performed using the Seoul Neuropsychological Screening Battery [2]. The results are summarized in table 1. Forward and backward digit span were markedly reduced. The Boston Naming Test showed severely impaired confrontational naming ability. Other language functions were intact. Gerstmann syndrome, buccofacial and ideomotor apraxia were observed. The Rey-Osterrieth Complex Figure Test revealed visuoconstructive dysfunction. The Seoul Verbal Learning Test and the Rey-Osterrieth Complex Figure Test showed that both verbal and visual memory functions were severely impaired. Frontal surveys revealed poor frontal executive function, generative naming abilities, and inhibitory control.

\section{Genetic Studies}

Genomic DNA isolated from peripheral blood leukocytes was used for analysis. Direct sequencing of the PRNP gene identified a heterozygous p.Asp179Asn mutation homozygous for methionine at codon 129 and for glutamate at codon 219.

\section{SPM Analysis of FDG-PET}

The patient's FDG-PET scan showed mild hypometabolism in the bilateral frontal cortices and bilateral thalamus (fig. 2a). Using SPM analysis, the patient's PET scan was compared with those of 7 healthy male controls (fig. 2b). The results of the SPM analysis showed marked hypometabolism in the patient's deep cerebral nuclei including the bilateral thalami, caudate nuclei, and hypothalamus. In addition, the hypometabolism affected association cortices including the frontal, lateral temporal, inferior parietal lobule, and posterior cingulate gyri; among these, frontal association areas including the dorsolateral, medial and orbitofrontal areas were more dominantly affected than the other lobes. Lastly, in addition to cerebral lesions, the midbrain was also affected.

\section{Discussion}

We presented a first Korean FFI family and an SPM analysis of brain FDG-PET of the index case. The patient's symptoms were compatible with FFI, the laboratory findings showed sympathetic hyperactivity supporting the diagnosis, and his genetic abnormality was diagnostic of FFI. In the patient's family, the affected members showed variable disease onset and symptoms with reduced penetrance, which is consistent with a previous report [3]. Interestingly, all of the suspected and confirmed FFI patients in this family were male, while the obligate female carriers did not present FFI-related signs or symptoms. So far, no specific sex dominance has been reported in previous studies of FFI kindred from different 
races and regions [1, 3-7]. Our present case suggests that the penetrance of FFI could be sexselective, at least in a specific kindred.

In the SPM study, the patient showed decreased glucose metabolism of the midbrain and the hypothalamus. In FDG-PET studies which reported cerebral glucose metabolism in FFI patients, thalamic hypometabolism was consistently found, even in presymptomatic stages $[8,9]$. However, brainstem and hypothalamic hypometabolism has seldom been reported. Along with the thalamus, brainstem reticular activation is important in the sleep-wake cycle, as the thalamus and brainstem are reported to be deactivated during slow-wave sleep. This finding is in line with pathological studies which have revealed that the midbrain contains comparable amounts of protease-resistant prion proteins to the thalamus, regardless of disease duration [10]. The hypothalamic hypometabolism demonstrated in our case may also have clinical implications. The hypothalamus plays a role in regulating sympathetic function, and thus it might be possible for prominent sympathetic instability and dysregulation of FFI to be related to hypothalamic dysfunction. Although Raggi et al. [9] reported an SPM analysis of a FFI case showing subthalamic hypometabolism, hypothalamic hypometabolism has not yet been described. Thus, the results of the SPM analysis of the present case could give new evidence showing that the midbrain and hypothalamus might have a role in the pathophysiology of FFI.

In conclusion, this is the first Korean report of an FFI patient whose family showed male phenotypic predominance. SPM analysis demonstrated brain hypometabolism in the midbrain and the hypothalamus in addition to various brain regions (thalami, caudate nuclei and multiple cortical regions) that have been reported in previous studies. Future studies involving more cases are needed.

\section{Acknowledgement}

This study was supported by a Samsung Medical Center Clinical Research Development Program grant (CRS110-14-1).

\section{Disclosure Statement}

The authors report no conflicts of interest.

\section{References}

1 Lugaresi E, Medori R, Montagna P, Baruzzi A, Cortelli P, Lugaresi A, et al: Fatal familial insomnia and dysautonomia with selective degeneration of thalamic nuclei. N Engl J Med 1986;315:997-1003.

$\rightarrow 2$ Ahn H-J, Chin J, Park A, Lee BH, Suh MK, Seo SW, et al: Seoul Neuropsychological Screening Battery-dementia version (SNSB-D): a useful tool for assessing and monitoring cognitive impairments in dementia patients. J Korean Med Sci 2010;25:1071-1076.

-3 Synofzik M, Bauer P, Schols L: Prion mutation D178N with highly variable disease onset and phenotype. J Neurol Neurosurg Psychiatry 2009;80:345-346.

-4 Almer G, Hainfellner JA, Brucke T, Jellinger K, Kleinert R, Bayer G, et al: Fatal familial insomnia: a new Austrian family. Brain 1999;122(Pt 1):5-16.

5 Harder A, Jendroska K, Kreuz F, Wirth T, Schafranka C, Karnatz N, et al: Novel twelve-generation kindred of fatal familial insomnia from Germany representing the entire spectrum of disease expression. Am J Med Genet 1999;87:311-316.

-6 Tabernero C, Polo JM, Sevillano MD, Munoz R, Berciano J, Cabello A, et al: Fatal familial insomnia: clinical, neuropathological, and genetic description of a Spanish family. J Neurol Neurosurg Psychiatry 2000;68:774777. 


\begin{tabular}{l|l}
\hline DOI: $10.1159 / 000365412$ & $\begin{array}{l}\text { C 2014 S. Karger AG, Basel } \\
\text { www.karger.com/crn }\end{array}$ \\
\hline
\end{tabular}

Lee et al:: Midbrain Hypometabolism in Fatal Familial Insomnia: A Case Report and a Statistical Parametric Mapping Analysis of a Korean Family

7 Spacey SD, Pastore M, McGillivray B, Fleming J, Gambetti P, Feldman H: Fatal familial insomnia: the first account in a family of Chinese descent. Arch Neurol 2004;61:122-125.

8 Cortelli P, Perani D, Montagna P, Gallassi R, Tinuper P, Federica P, et al: Pre-symptomatic diagnosis in fatal familial insomnia: serial neurophysiological and 18FDG-PET studies. Brain 2006;129:668-675.

$\checkmark 9$ Raggi A, Perani D, Giaccone G, Iannaccone S, Manconi M, Zucconi M, et al: The behavioural features of fatal familial insomnia: a new Italian case with pathological verification. Sleep Med 2009;10:581-585.

10 Parchi P, Castellani R, Cortelli P, Montagna P, Chen SG, Petersen RB, et al: Regional distribution of proteaseresistant prion protein in fatal familial insomnia. Ann Neurol 1995;38:21-29. 

function

\begin{tabular}{|c|c|c|}
\hline Tests & $\begin{array}{l}\text { Raw score/ } \\
\text { total score }\end{array}$ & $\begin{array}{l}\text { Percentile } \\
\text { score }\end{array}$ \\
\hline K-MMSE & $17 / 30$ & 0.01 \\
\hline \multicolumn{3}{|l|}{ Attention } \\
\hline Digit span: forward & $4 / 9$ & 1.66 \\
\hline Digit span: backward & $2 / 8$ & 3.92 \\
\hline \multicolumn{3}{|l|}{ Language } \\
\hline Spontaneous speech & nonfluent & \\
\hline Comprehension & normal & \\
\hline Repetition & $15 / 15$ & $\geq 16$ \\
\hline $\begin{array}{l}\text { Naming: short version of the K- } \\
\text { BNT }\end{array}$ & $4 / 15$ & 0.01 \\
\hline Reading & normal & \\
\hline Writing & normal & \\
\hline \multicolumn{3}{|l|}{ Gerstmann syndrome and praxis } \\
\hline Finger naming & borderline & \\
\hline Right-left orientation & normal & \\
\hline Body part identification & abnormal & \\
\hline Calculation & $12 / 12$ & $\geq 16$ \\
\hline Praxis: ideomotor & $2 / 5$ & $<16$ \\
\hline Praxis: buccofacial & abnormal & \\
\hline \multicolumn{3}{|l|}{ Visuospatial functions } \\
\hline Rey Complex Figure Test copy & $26.5 / 36$ & 0.01 \\
\hline \multicolumn{3}{|l|}{ Memory } \\
\hline SVLT: immediate recall & $4 / 36$ & 0.01 \\
\hline SVLT: delayed recall & $0 / 12$ & 0.29 \\
\hline SVLT: recognition score & $12 / 24$ & 1.29 \\
\hline RCFT: immediate recall & $0.5 / 36$ & 0.28 \\
\hline RCFT: delayed recall & $0 / 36$ & 0.34 \\
\hline RCFT: recognition score & $12 / 24$ & 1.29 \\
\hline \multicolumn{3}{|l|}{ Frontal/executive functions } \\
\hline Motor impersistence & normal & \\
\hline Contrasting program & $20 / 20$ & $\geq 16$ \\
\hline Go/no go & $14 / 20$ & $<16$ \\
\hline Fist-edge-palm & normal & \\
\hline Alternating hand movement & abnormal & \\
\hline Alternating square and triangle & perseveration & \\
\hline Luria loop & perseveration & \\
\hline COWAT: animal & 1 & 0.04 \\
\hline COWAT: supermarket & 1 & 0.04 \\
\hline COWAT: phonemic total score & 5 & 0.06 \\
\hline Stroop test: word reading & $112 / 112$ & $\geq 16$ \\
\hline Stroop test: color reading & $23 / 112$ & 0.01 \\
\hline
\end{tabular}

K-MMSE $=$ Korean version of the Mini-Mental State Examination; K-BNT = Korean version of the Boston Naming Test; RCFT = ReyOsterrieth Complex Figure Test; SVLT = Seoul Verbal Learning Test; COWAT $=$ Controlled Oral Word Association Test. 
Lee et al:: Midbrain Hypometabolism in Fatal Familial Insomnia: A Case Report and a Statistical Parametric Mapping Analysis of a Korean Family

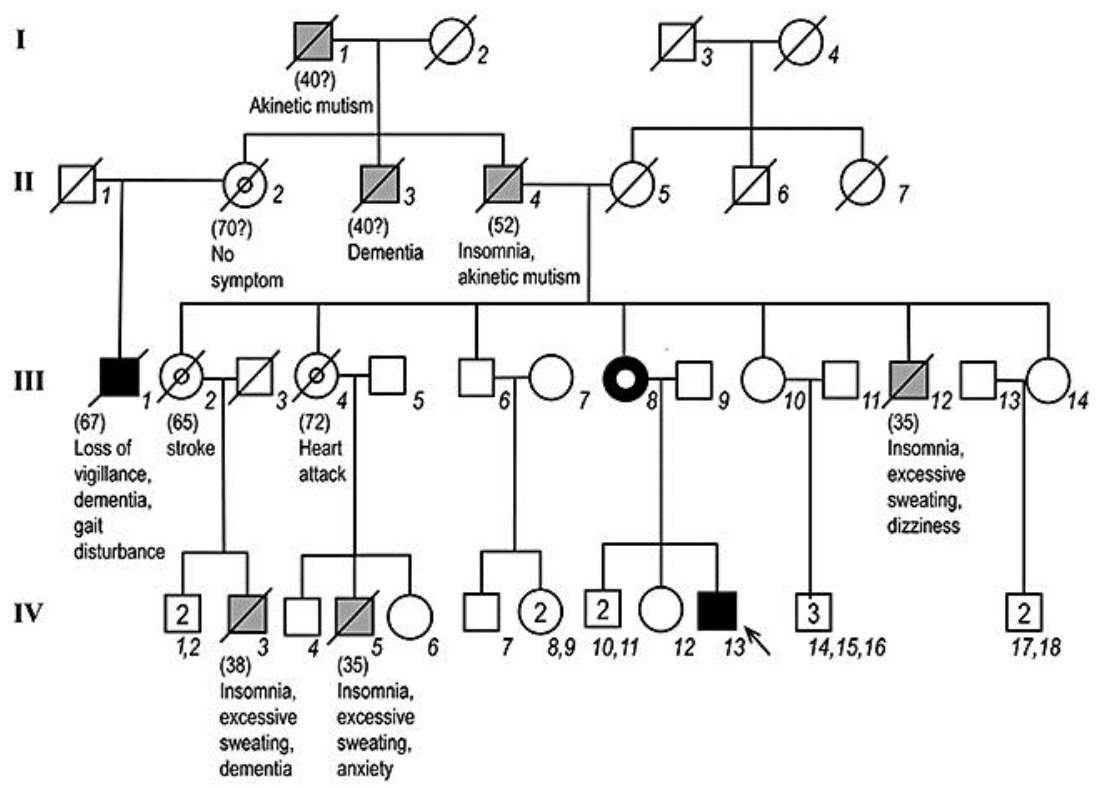
Female
$\square$ Male
(2) Possible carrier
$\square$ Possible FFI according to history
Genetically confirmed carrier
Definite FFI confirmed by genetic study

Fig. 1. The pedigree of a Korean family with FFI. The diagonal bar indicates a deceased member. Arrow = The proband. 


\section{Case Reports in Neurology}

\begin{tabular}{l|l}
\hline \multicolumn{2}{l}{ Case Rep Neurol 2014;6:243-250 } \\
\hline DOI: $10.1159 / 000365412$ & $\begin{array}{l}\text { C 2014 S. Karger AG, Basel } \\
\text { www.karger.com/crn }\end{array}$ \\
\hline
\end{tabular}

Lee et al:: Midbrain Hypometabolism in Fatal Familial Insomnia: A Case Report and a Statistical Parametric Mapping Analysis of a Korean Family
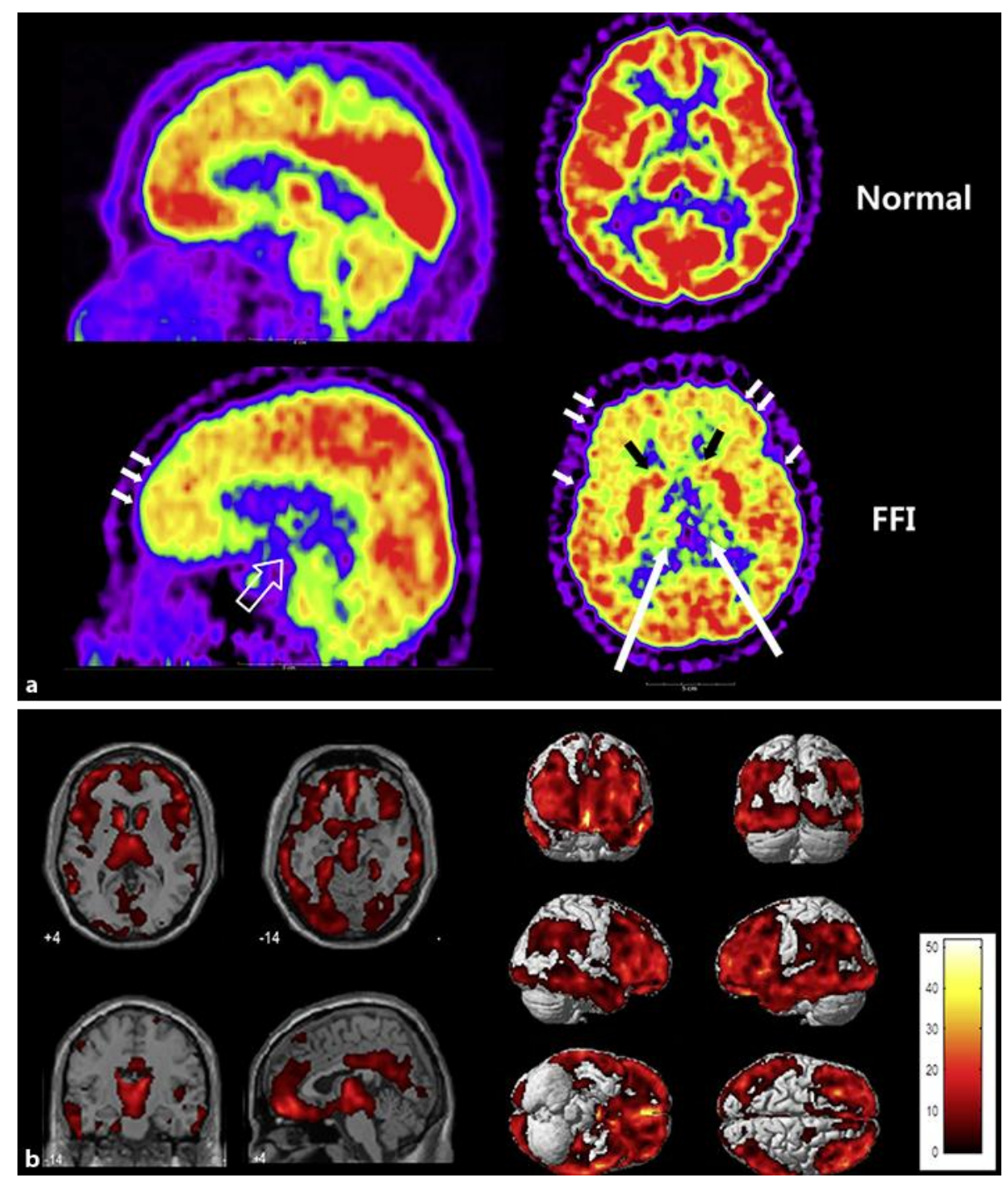

Fig. 2. SPM analysis of the patient's $18 \mathrm{~F}-\mathrm{FDG}$ brain PET. a The proband's ${ }^{18} \mathrm{~F}-\mathrm{FDG}$ brain PET scan shows hypometabolism of the bilateral thalami (long arrows), caudate nuclei (black arrows), midbrain (empty arrow), and frontal and temporal cortices (short arrows). b SPM analysis of the proband. Prominent hypometabolism was observed in the bilateral caudate nuclei, thalami, hypothalamus, frontotemporal cortices, posterior cingulate gyri, precuneus and midbrain. Colored areas indicate significantly decreased metabolism compared with 7 healthy male controls (FDR-corrected $\mathrm{p}<0.05$ ). 